\title{
Mapping Mt. Ushba: Preparation for a new Alpine Club Map
}

\author{
Mathias Gröbe*, Nikolas Prechtel, Benjamin Schröter, Dirk Burghardt
}

TU Dresden, Institute of Cartography, mathias.groebe@tu-dresden.de,nikolas.prechtel@tu-dresden.de, benjamin.schroeter@tudresden.de,dirk.burghardt@tu-dresden.de

* Corresponding author

Keywords: map making, fieldwork, data collection, data evaluation, LBSN

\begin{abstract}
:
Mt. Ushba is situated in the Caucasus that forms the border between Georgia and Russia. On the Georgian side the historical province of Svaneti is located, famous for its spectacular mountains, botany, and tower houses, which are part of the UNESCO World Heritage Sites. In addition, Svaneti is a popular touristic destination in winter and summertime. In cooperation with the German Alpine Club, the Institute of Cartography of Dresden University of Technology decided to create a new large-scale Alpine Club map for this area. Target is a high-quality map for various mountain activities in the Alpine Club tradition, along with sustainable data storage, multi-source evaluation, and reuse of geo-information of existing topographic references and OpenStreetMap. A further important information source will be remote sensing imagery provided by Planet Labs Inc. As a result of our field campaign many newly captured and updated data will be made available in return in OpenStreetMap. Existing hiking apps, which usually base upon OpenStreetMap, will therefore also benefit from our work
\end{abstract}

First, we looked at the existing maps and available geo-data of the region, which may serve as a basis for mapping activities or evaluation. So far, we could identify the following sources: topographic and touristic maps at scales between 1:25,000 and 1:50,000, OpenData, OpenStreetMap, Location-based Social Media (LBSM) (mainly photos), satellite imagery, and Digital Elevation Models (e.g. SRTM30). Building a visualization on top of collected data gives a first impression of the of the region to be mapped. It reveals touristic hotspots and frequently used trails, but also rarely visited areas. In combination with travel guides this visualization formed a reasonable basis for two initial decisions: The tailoring of the map extent and a precise planning of routes, where a collection and validation of geo-data shall be carried out in the field.

Due to the COVID-19 pandemics, one could not realise the fieldwork as planed in 2020. However, we tried to prepare for the expedition in 2021 as intense as possible by office work, starting with data preparation and data generation based on satellite images and OpenStreetMap data. Master theses were initiated on the topics of image classification of vegetation, rock, and ice and modes of geo-data acquisition in order to improve the OpenStreetMap database. Another source is old and up-to-date topographic maps. They help to estimate the integrity of the OpenStreetMap data. The information service of libraries, e.g., the FID ${ }^{1}$ has helped us to find and to digitize relevant existing map content. The scanned versions were georeferenced and integrated with the other digital data.

A WMS and WFS service was set up to integrate all data for further processing. It facilitates, and centralises data storage. Data management and continuous updates become easier. The OpenStreetMap data was stored in a PostgreSQL/PostGIS database and visualized in the WMS by QGIS as a dynamic draft version and a field mapping basis. At the ICC conference experiences of the field campaign shall be presented. Field mapping will take place in July and August 2021. Mapping techniques and challenges will be explained along with methods of data processing and upgrading for the production of the Alpine Club map covering the region Ushba/Mestia.

\footnotetext{
${ }^{1}$ https://kartographie.staatsbibliothek-berlin.de/
} 